\title{
Effect of the vacancy interaction on antiphase domain growth in a two-dimensional binary alloy
}

\author{
Marcel Porta, Carlos Frontera, Eduard Vives, and Teresa Castán \\ Departament d'Estructura i Constituents de la Matèria, Facultat de Física, Universitat de Barcelona, Diagonal 647, \\ E-08028 Barcelona, Catalonia, Spain
}

(Received 26 March 1997; revised manuscript received 16 May 1997)

\begin{abstract}
We have performed a Monte Carlo simulation of the influence of diffusing vacancies on the antiphase domain growth process in a binary alloy after a quench through an order-disorder transition. The problem has been modeled by means of a Blume-Emery-Griffiths Hamiltonian whose biquadratic coupling parameter $K$ controls the microscopic interactions between vacancies. The asymmetric term $L$ has been taken as $L=0$ and the ordering dynamics has been studied at very low temperature as a function of $K$ inside the range $-0.5 \leqslant K / J \leqslant 1.40$ (with $J>0$ being the ordering energy). The system evolves according to the Kawasaki dynamics so that the alloy concentration is conserved while the order parameter is not. The simulations have been performed on a two-dimensional square lattice and the concentration has been taken so that the system corresponds to a stoichiometric alloy with a small concentration of vacancies. We find that, independently of $K$, the vacancies exhibit a tendency to concentrate at the antiphase boundaries. This effect gives rise, via the vacancy-vacancy interaction (described by $K$ ), to an effective interaction between bulk diffusing vacancies and moving interfaces that turns out to strongly influence the domain growth process. One distinguishes three different behaviors: (i) For $K / J<1$ the growth process of ordered domains is anisotropic and can be described by algebraic laws with effective exponents lower than $1 / 2$; (ii) $K / J \simeq 1$ corresponds to the standard Allen-Cahn growth; (iii) for $K / J>1$ we found that, although the motion of the interface is curvature driven, the repulsive effective interaction between both the vacancies in the bulk and those at the interfaces slows down the growth. [S0163-1829(97)06933-6]
\end{abstract}

\section{INTRODUCTION}

The dynamical evolution of a binary alloy after a quench from a high-temperature disordered phase has been one of the prototypes in the study of relaxational processes towards equilibrium. It has been found that the late stages of this process obey dynamical scaling and the typical domain size $R(t)$ dominates all other lengths. ${ }^{1,2}$ In this regime, the domains grow in time according to a power law $R(t) \sim t^{x}$ with the growth exponent $x$ satisfying a remarkable universality. For the case of a pure, ideal system, the exponent $x=1 / 2$ is associated with cases where the order parameter is not conserved, ${ }^{3}$ whereas $x=1 / 3$ describes systems with conserved order parameter. ${ }^{4}$ For the nonconserved case a typical example is a binary alloy undergoing an order-disorder phase transition. Most theoretical studies are limited to ideal conditions, that is, to a pure stoichiometric binary alloy. In such conditions, theory ${ }^{3}$ and numerical simulations ${ }^{5-8}$ definitively agree about the value of the kinetic exponent $x=1 / 2$. Much less unanimity is obtained from the experiments. This is certainly due to the imperfections always present in real materials. Some examples are vacancies, third-component impurities, ${ }^{9}$ nonstoichiometry, ${ }^{10}$ dislocations, etc. It is of great interest to elucidate in which manner and to what extent the presence of these imperfections modifies the ideal asymptotic growth law.

We shall not consider here the problem of quenched disorder, ${ }^{11}$ but concentrate on mobile punctual defects such as vacancies, third-component impurities, and excess particles in off-stoichiometric binary alloys. These belong to the category commonly named annealed disorder. Despite the theoretical suggestion that such a kind of disorder should not modify the asymptotic growth law, ${ }^{12}$ experiments, ${ }^{10}$ and nu- merical simulation studies ${ }^{12-15}$ provide evidences for slow growth, either logarithmic growth laws or algebraic laws with small exponents.

A general feature commonly observed during the earlytime evolution in systems with annealed defects is the tendency to concentrate the disorder at the domain walls. Indeed, experiments ${ }^{10,16}$ and numerical simulations ${ }^{8,12-15,17}$ reveal that the vacancies and the excess particles tend to accumulate at the domain walls. This is accompanied by a depletion in the bulk defect concentration, which renders the excess internal energy unsuitable to measure the total amount of interfaces. Only at late times, as the interfaces disappear and the system approaches equilibrium, the annealed defects may dissolve again into the bulk, provided that they display no cooperative phenomena. Simultaneously an overshooting in the bulk order parameter is observed. ${ }^{12,18}$ Very recently it has been suggested ${ }^{12,18,19}$ that this is a generic effect in ordering dynamics, coming from a subtle competition between nonequilibrium internal energy and nonequilibrium entropy.

In previous ${ }^{13,14}$ studies of the diluted square Ising model with nearest-neighbor interactions, it was obtained that the effect of a small concentration of vacancies is dramatic, leading to an extremely slow growth described by a logarithmic growth-law ${ }^{14}$ or even to a complete pinning of the process. ${ }^{13}$ This difference, obtained on the same model in the limit of low vacancy concentration, comes from special features introduced in the coupled dynamics used; that is, the system evolves according to the nonconserved Glauber dynamics but the vacancy concentration is forced to be constant. We notice that in these studies, the vacancies exhibit a natural tendency to cluster. The results obtained in the present work show that the true asymptotic growth behavior is definitively 
algebraic with effective exponents smaller than the AllenCahn value.

Concerning the present scenario for the effect of excess particles in a nonstoichiometric binary alloy without vacancies, very recently it was suggested ${ }^{8}$ that the existence of effective interactions between diffusing excess particles and those localized at the antiphase domain boundaries is crucial in determining the essential time dependence of the growth law. It was shown that when these specific interactions are not present the main assumptions underlying the Allen-Cahn theory are fulfilled. On the other hand there is experimental evidence that small deviations from the stoichiometric composition may provoke drastic modifications in the growth law. It has been reported that the ordering kinetics in $\mathrm{Cu}_{0.79} \mathrm{Au}_{0.21}{ }^{10}$ shows a crossover from the standard AllenCahn growth law, for stoichiometric $\mathrm{Cu}_{3} \mathrm{Au},{ }^{10,20}$ to a logarithmic growth law. In Ref. 8 it was suggested that to account for such behavior, additional interactions to the ones present in the (nearest neighbors) Ising model are needed. Indeed, the authors showed how this can be accomplished by simply extending the interactions to next nearest neighbors. Our main interest here will be to incorporate the effect of such interactions between annealed impurities in a more general framework.

The three-state Blume-Emery-Griffiths (BEG) model is especially adequate for our purposes. ${ }^{21}$ For given values of the model parameters $(K$ and $L)$, the third value of the spin variable may represent either a vacancy or an impurity (in particular an excess particle). In the present work we neglect the asymmetric term $(L=0)$ and restrict ourselves to the case of vacancies in a stoichiometric binary alloy, so that the additional coupling coming from the interplay between the diffusive motion of both vacancies and excess particles is not considered here.

The influence of mobile vacancies on the kinetics of ordering arises from the coupling between their diffusive dynamics and the motion of the domain walls [in this case, antiphase-domain boundaries (APB)]. This intercoupling depends on the two following facts concerning the behavior of the vacancies: their tendency to precipitate at the APBs and their tendency to cluster. Whereas the former is encountered for all values of the model parameters studied in this work, the interaction among vacancies, and furthermore their tendency to cluster, depends on $K$. The combination of both effects gives rise to an effective interaction (controlled by $K$ ) between bulk diffusing vacancies and those localized at the interfaces that turns out to be crucial in determining the essential time dependence of the growth law.

The organization of this paper is the following. We start by defining the model and the region of parameters of interest here (Sec. II). In Sec. III we provide the details of the simulations and describe the algorithms used. In Sec. IV we present the results and discuss them in Sec. V. Finally, in Sec. VI we summarize our main conclusions.

\section{THE MODEL AND PARAMETERS}

We assume an underlying rigid square lattice with $i=1, \ldots, N=l \times l$ sites that can be occupied either by $A$ atoms $\left(S_{i}=1\right), B$ atoms $\left(S_{i}=-1\right)$, or vacancies $\left(S_{i}=0\right)$. Following standard procedures, the interactions are taken to be
TABLE I. Bond energies and the same measured with respect to the $A-B$ bond for the BEG model as a function of the parameters $J$, $K$, and $L$.

\begin{tabular}{lcc}
\hline \hline Bond & Energy & Excess energy \\
\hline$A-B$ & $-J+K$ & 0 \\
$A-A$ & $J+K+2 L$ & $2 J+2 L$ \\
$B-B$ & $J+K-2 L$ & $2 J-2 L$ \\
$A-V$ & 0 & $J-K$ \\
$B-V$ & 0 & $J-K$ \\
$V-V$ & 0 & $J-K$ \\
\hline \hline
\end{tabular}

pair wise and restricted to nearest neighbors (n.n.) only. The spin-1 BEG Hamiltonian is

$$
\mathcal{H}=J \sum_{i, j}^{\text {n.n. }} S_{i} S_{j}+K \sum_{i, j}^{\text {n.n. }} S_{i}^{2} S_{j}^{2}+L \sum_{i, j}^{\text {n.n. }}\left(S_{i}^{2} S_{j}+S_{i} S_{j}^{2}\right),
$$

where $J$ stands for the atom-atom ordering interaction, $K$ is a biquadratic coupling parameter accounting for the energy difference between atom-atom pairs and those involving vacancies, and $L$ is an asymmetry term accounting for the energy difference between $A-A$ and $B-B$ pairs. When the parameter $K$ promotes the formation of atom-atom pairs, the vacancies tend to cluster, whereas if the vacancy-atom pairs are preferred, cooperative effects for the vacancies are not expected, at least in the limit of low vacancy concentration.

Although the hypothesis of a rigid lattice is crude, since lattice deformations have a strong influence on the atom dynamics around the vacancies, this effect is partially taken into account in the phenomenological character of the constant $K$. We also expect that the assumption of pair interactions only, disregarding many-body effects, only introduces quantitative but not qualitative changes concerning the ordering dynamics.

We have restricted the present study to concentrations so that the system corresponds to a stoichiometric binary alloy with small concentration of vacancies. Since we have taken $J>0$, the ordered phase will be antiferromagneticlike, with almost all the bonds of the $A-B$ kind.

As we have mentioned in the Introduction our goal here is to study the influence of annealed vacancies on the kinetics of domain growth. This influence originates in the interplay between the two following specific interactions: (i) the vacancy-APB interaction (here the APB is thought as a sequence of $A-A$ and $B-B$ bonds) and (ii) the vacancy-vacancy interaction. When the the vacancy-APB interaction is attractive, the vacancies tend to concentrate at the APBs and therefore the vacancy-vacancy interaction introduces an effective interaction between bulk vacancies and APBs.

The next step is to calculate these specific interactions for the different values of the model parameters. They are obtained from the bond energies, expressed with respect to the energy of the $A-B$ bond, summarized in Table I. For notation we introduce the following reduced parameters $K^{*}=K / J$, $L^{*}=L / J$, with $J>0$. It follows that $K^{*}=1$ separates the tendency for the vacancies to cluster $\left(K^{*}<1\right)$ or not $\left(K^{*}>1\right)$. Moreover, for $\left|L^{*}\right|<1$ the specific interaction between vacancies and APBs is attractive, favoring the absorption of vacancies at the interfaces. For values of $\left|L^{*}\right|>1$ the 


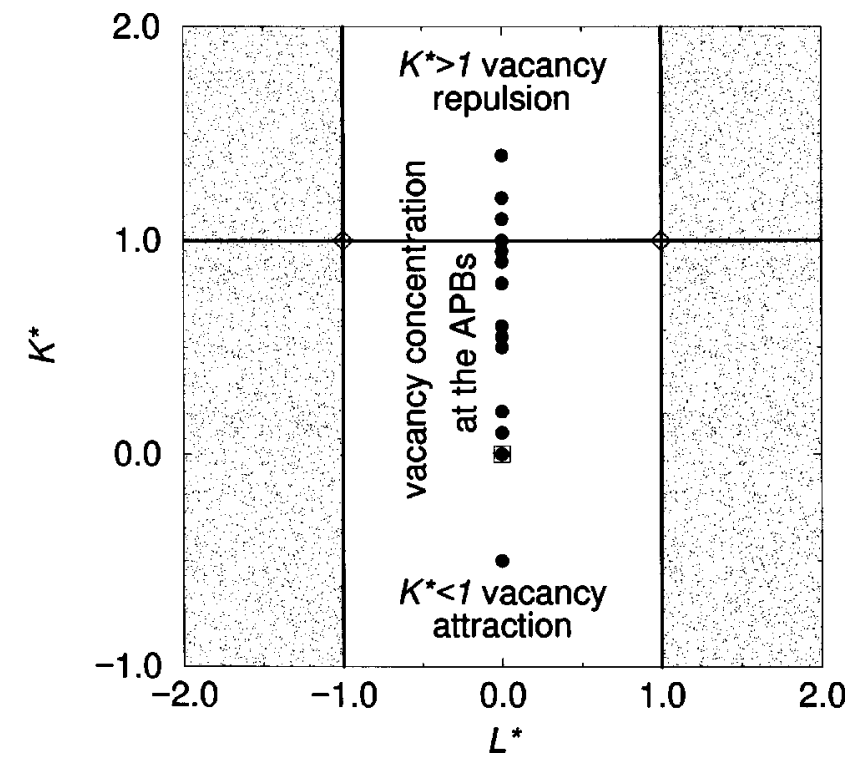

FIG. 1. Regions in the space of the parameters $K^{*}$ and $L^{*}$ where different dynamics are expected. The solid line $K^{*}=1$ separates the region of vacancy attraction $\left(K^{*}<1\right)$ from the region of vacancy repulsion $\left(K^{*}>1\right)$. The solid lines $L^{*}= \pm 1$ separate the region where the vacancies tend to precipitate at the antiphase boundaries $\left(\left|L^{*}\right|<1\right)$ from the region of vacancy-antiphase boundary repulsion $\left(\left|L^{*}\right|>1\right)$. Solid circles indicate the points studied in the present work. The square corresponds to the case $K^{*}=L^{*}=0$ and the diamonds are the points where the model is formally equivalent to a nonstoichiometric binary alloy.

behavior is more complex, in particular, if $K^{*}<1$ a competition between $K^{*}$ and the asymmetric term $L^{*}$ appears.

The results of the above analysis are illustrated in Fig. 1. We have indicated, in white, the region where the vacancies exhibit tendency to accumulate at the APBs. The line $K^{*}=1$ separates the regions with vacancy attraction and vacancy repulsion. Black circles indicate the points studied in the present work, all sitting along the line $L^{*}=0$. The point at $K^{*}=0$ and $L^{*}=0$ (indicated by a square) has been previously studied in $^{14}$ and corresponds to a diluted Ising model. The points with $K^{*}=1$ and $L^{*}= \pm 1$, indicated by diamonds, correspond to a nonstoichiometric binary alloy without vacancies. ${ }^{8}$

It is worth mentioning that the BEG model, with $K=0$, $L=0$, and $J>0$, has also been used for the study of the ordering dynamics via vacancies with $c_{V}<4 \times 10^{-4}$. Such a restricted dynamics, only allowing exchanges between atoms and vacancies, may strongly modify the dynamics. ${ }^{22}$ This mechanism is not considered in the present study.

\section{MONTE CARLO SIMULATIONS}

We have performed different Monte Carlo simulations of the model defined in Sec. II with $L^{*}=0, J>0$, and $K^{*}$ inside the range $-0.5 \leqslant K^{*} \leqslant 1.4$. First we need to know, in the region of vacancy attraction $\left(K^{*}<1\right)$, the temperature at which the system separates into two phases. This is important in order to characterize the equilibrium state at the point to which quenches have been performed. Next, in order to characterize the time evolution of the ordering process subsequent to the quench, we focus on the study of the structure
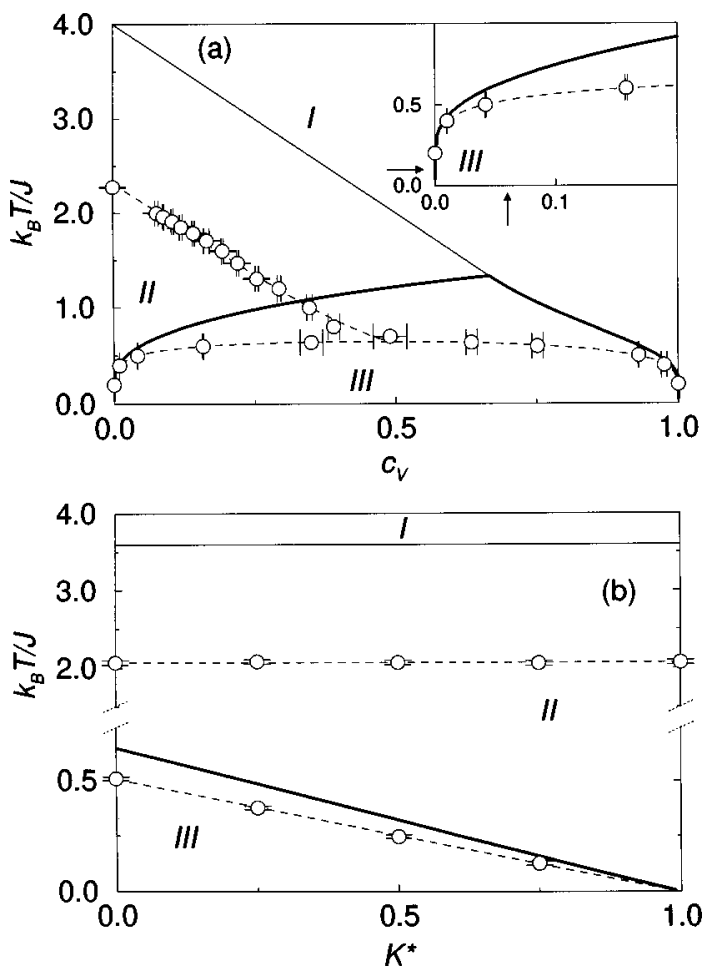

FIG. 2. (a) $k_{B} T / J$ vs $c_{V}$ phase diagram of the BEG model for $K^{*}=L^{*}=0$. (b) $k_{B} T / J$ vs $K^{*}$ phase diagram of the BEG model for $L^{*}=0$ and $c_{V}=0.06$. The points correspond to the Monte Carlo results whereas the solid lines are obtained from mean-field calculations. Dashed lines are just guides to the eyes. In the inset, we show the region of interest. The arrows indicate the working temperature and vacancy concentration

factor width. It is known that the structure factor provides an overall description of the ordering process. In particular, the study of its time evolution will provide information about the dynamical scaling properties. This second group of simulations constitutes the major part of results presented and, contrarily to the equilibrium simulations, is very time consuming.

\section{A. Equilibrium simulations: Phase diagram}

In order to obtain the phase diagram of model (1) in the particular case of $L^{*}=0$, we have performed Monte Carlo simulations in the grand canonical ensemble using the Legendre transformation

$$
\mathcal{H}_{\mathrm{GC}}=\mathcal{H}-\mu \sum S_{i}^{2}
$$

where $\mu$ stands for the chemical potential difference between atoms (either $A$ or $B$ ) and vacancies. This is because we restrict our study to the case of stoichiometric composition $\left(N_{A}=N_{B}\right)$ and the chemical potentials of $A$ and $B$ are then equal. The simulations have been performed on a system of linear size $l=128$ using the Glauber dynamics implemented into the Metropolis algorithm. The different runs are extended up to 1500 Monte Carlo steps per site (MCs), our unit of time.

Figures 2(a) and 2(b) show two different sections of the phase diagram obtained by Monte Carlo simulations. In the 
same figure we also show the results obtained by using standard mean-field techniques (self-consistent field method). ${ }^{23}$ This has been done for completeness and in order to reduce the range of model parameters to be explored numerically. Figure 2(a) (temperature versus vacancy concentration) corresponds to a section with fixed $K^{*}=0$, and Fig. 2(b) (temperature versus $\left.K^{*}\right)$ to a section with fixed vacancy concentration $c_{V}=0.06$. Phase I corresponds to an atomic disordered phase with randomly diluted vacancies; phase II corresponds to an atomic ordered $A B$ phase with randomly diluted vacancies, which exhibit only short-range order; phase III corresponds to a phase separation region with coexisting ordered $A B$ domains with low concentration of vacancies and vacancy clusters with a low concentration of disordered atoms. The inset in Fig 2(a) shows an enlarged portion of the coexistence region in the limit of very low vacancy concentration. In this limit both phase transitions $(\mathrm{I} \rightarrow \mathrm{II}$ and $\mathrm{II} \rightarrow \mathrm{III})$ are very separated, so that they occur almost independently. This is reflected in the straight-lineshaped boundaries in Fig 2(b). One obtains that the orderdisorder transition temperature $(\mathrm{I} \rightarrow \mathrm{II})$ is independent of $K^{*}$, while the temperature for the phase separation transition (II $\rightarrow$ III) depends linearly on $K^{*}$. We expect that for higher values of $c_{V}$ the coupling between both phase transitions makes the boundaries become curved. In any case notice that, when $K^{*}>1$, the coexistence region (III) disappears, and that the $A B$ ordered phase (II) extends down to $T=0$.

\section{B. Nonequilibrium simulations}

Although most of the results, presented in the next section, have been obtained following the standard Kawasaki dynamics, alternative optimized algorithms have been used when specially long simulations were needed. This subsection is devoted to a description of the different algorithms used in the study of the time evolution of the process that follows a thermal quench from very high temperature (disordered phase) to $T=0.1 J / k_{B}$ performed on a stoichiometric binary alloy with a small concentration of vacancies fixed at $c_{V}=0.06$ (being $c_{A}=c_{B}=0.47$ ). The different values of $K^{*}$ studied correspond to final states into the ordered phases either II and III.

\section{Standard dynamical simulations}

These are simulations performed using the standard Metropolis algorithm together with the Kawasaki dynamics. The linear system size is $l=200$ even though some initial studies were performed on systems of $l=100$. Starting from an initial disordered configuration, the runs have been (typically) extended up to $20000 \mathrm{MCs}$. Moreover, averages over about 20 independent realizations have been performed. From each simulation we have extracted the time evolution of the structure factor defined as

$$
S(\vec{k})=\left|\frac{1}{N_{i}} \sum_{i=1, N} S_{i} \exp \left\{\mathrm{i} \frac{2 \pi}{a} \vec{k} \vec{r}_{i}\right\}\right|^{2},
$$

where $\vec{k}$ are the reciprocal space vectors, $a$ is the lattice parameter, and $\vec{r}_{i}$ is the vector position of site $i$. We have focused on the profiles along the (10) and (11) directions around the superstructure peak at $\vec{k}=\left(\frac{1}{2} \frac{1}{2}\right)$. Moreover, they have been averaged over equivalent directions. The size and shape of the ordered domains has been obtained by fitting the averaged profiles to a Lorentzian function powered to $3 / 2$ in order to reproduce the Porod's law for the decay of the tail at long $q$ 's: ${ }^{24}$

$$
S(q, t)=\left\{\frac{a(t)}{1+[q / \sigma(t)]^{2}}\right\}^{3 / 2}
$$

where $q$ is the distance to the superstructure peak $q=\left|\vec{k}-\left(\frac{1}{2}, \frac{1}{2}\right)\right|, a(t)$ is the height of the peak, and $\sigma(t)$ its width. Only data with $S(q, t)>S_{0}=2.5 \times 10^{-5}$ has been considered for the fits. $S_{0}$ has been obtained from a completely disordered system with the same concentration of particles and vacancies. The quantities $a(t)$ and $\sigma(t)$ provide information about the square order parameter growth and the inverse domains size, respectively.

\section{2. $N$-fold way algorithm}

For the particular case of $K^{*}=0$ and $L^{*}=0$ we have used the $N$-fold algorithm ${ }^{25}$ in order to reach very long times $\left(10^{7}\right.$ MCs) in the evolution of a system of linear size $l=200$. The possible exchanges have been classified into 11 different classes, according to their energy change. A class including those exchanges not modifying the system configuration has also been taken into account. This is done in order to compare with the standard dynamical simulations in which such exchanges are considered. The time elapsed after each exchange has been taken as the average time needed, in a standard Monte Carlo simulation, for the acceptance of a useful proposed exchange. In this case, the structure factor evolution has also been studied. The averages have been performed over $\sim 30$ independent runs.

\section{Optimized multigrid algorithm}

Due to the very large number of classes for $K^{*} \neq 0$ the $N$-fold algorithm becomes difficult to construct. Therefore, a simpler but less optimized algorithm has been constructed in order to reach very long times. Starting from a standard multigrid algorithm ${ }^{8,14}$ we have made, for each sublattice, a list of the exchanges whose probability of being accepted is not negligible. When a sublattice is chosen, only the exchanges present in the list are attempted. The method turns out to be very efficient when the number of attempted exchanges is low. In particular, for the case $K^{*}=0.6$ and $L^{*}=0$, we have performed simulations up to $10^{7} \mathrm{MCs}$ with no major difficulties (the system size was $l=200$ ).

\section{DOMAIN GROWTH RESULTS}

Figure 3 shows snapshots of the microconfigurations as they evolve with time after the quench for three different values of the parameter $K^{*}(=0.6,1.0$, and 1.4). The system size is $l=200$ and the quench temperature $T=0.1 J / k_{B}$. In this case we have used the standard dynamical algorithm described before. The vacancies are indicated in black while ordered regions are indicated in white. Due to the tendency exhibited by the vacancies to concentrate at the interfaces, the antiphase domain structure naturally shows up. The process of absorption of vacancies at the interfaces starts at very 

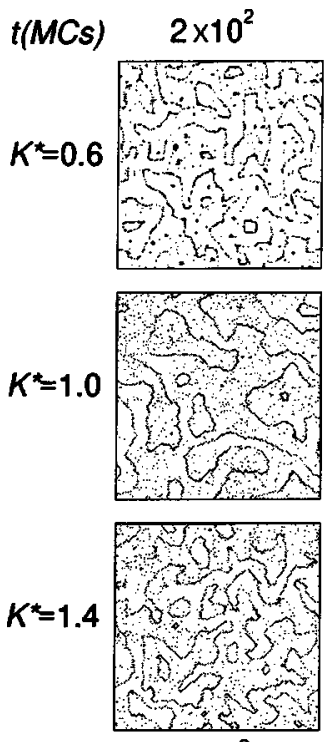

$2 \times 10^{2}$
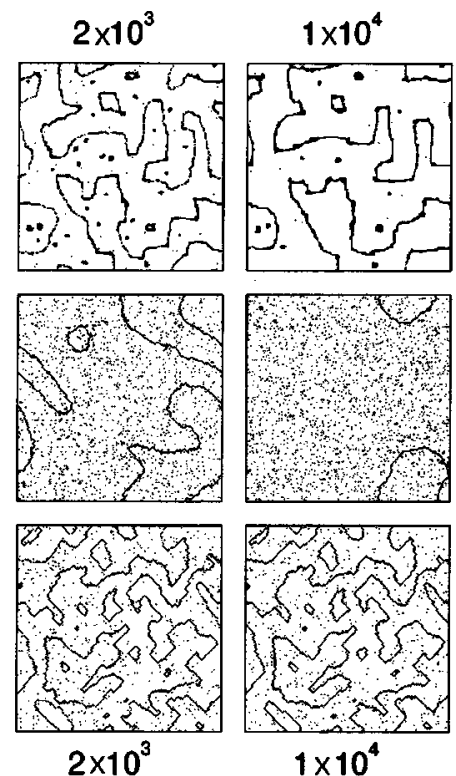

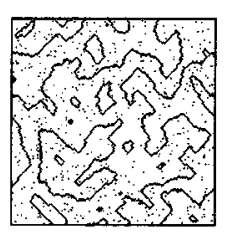

$1 \times 10^{4}$
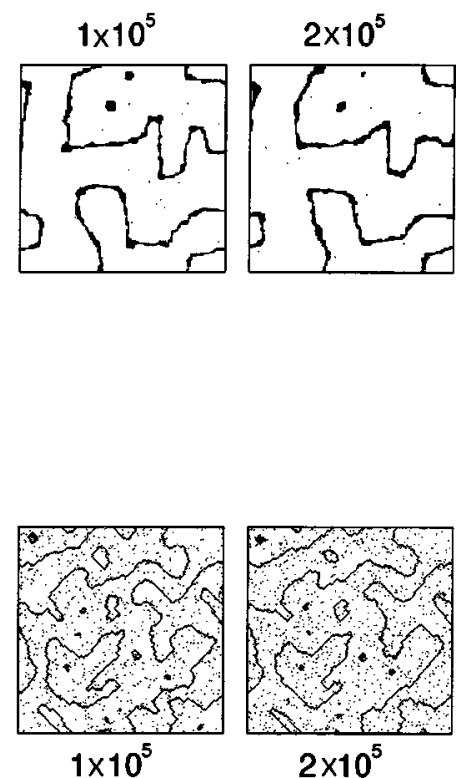

FIG. 3. Snapshots of the evolving domain structure for $K^{*}=0.6, K^{*}=1.0$, and $K^{*}=1.4$. Vacancies ( $A$ and $B$ particles) are painted in black (white). The simulations are performed in a $200 \times 200$ square lattice with $c_{V}=0.06$ at $T=0.1 J / k_{B}$. early times and follows until the whole interface network saturates. This initial regime was studied previously ${ }^{8}$ for the case of a nonstoichiometric binary alloy. Simultaneously, it was discussed in a more general context and suggested to be a generic effect in ordering dynamics. ${ }^{12,18,19}$ In any case, this is a transient, prior to the long-time domain growth regime of interest here. Nevertheless, we remark that this phenomenon of vacancy precipitation at the interfaces results in crucial importance in the subsequent evolution (dictated by interface reduction) specially when the interaction between vacancies is switched on $\left(K^{*} \neq 1\right)$. We now come back to Fig. 3. Clear differences can be observed in relation to both the orientation of the the antiphase boundaries and the speed of the evolution towards equilibrium. For $K^{*}=0.6$ (vacancy attraction), the domains appear squarelike with the interfaces preferably directed along the (10) direction. In the case $K^{*}=1.4$ (vacancy repulsion), although the domains are also square shaped, the interfaces are directed along the (11) direction. Moreover, in both cases, the interfaces tend to be flat (or almost flat), at least in the regime depicted in Fig. 3. As we shall discuss below, this is a consequence of the vacancyvacancy interaction that introduces energy barriers for the motion of the vacancies localized at the interfaces favoring, in each case, the different orientation of the APBs. Contrarily, no preferred orientation for the boundaries is observed when $K^{*}=1$. Remember that in this case there is no specific interaction between vacancies. Concerning the speed of the different evolutions, the fastest process occurs for $K^{*}=1$, whereas for the other two cases it is clearly slower, apparently even more for $K^{*}=1.4$. The introduction of specific interactions between vacancies seems to be behind the slower evolutions, although the underlying physics is, in both cases, different.

Before proceeding further it is interesting to look at the quantitative results obtained from structure factor calculations. In Fig. 4 we show the time evolution of the peak width $\sigma(t)$ of the structure factor along the two relevant directions, (10) (open circles) and (11) (filled circles) for the same three selected values of $K^{*}$ as in Fig. 3. Dashed lines indicate the regimes of algebraic domain growth and the numbers on top are the corresponding fitted values of the kinetic exponents.
For the case $K^{*}=1$ the algebraic growth breaks down before $\sim 10^{4}$ MCs due to finite-size effects. This is consistent with the snapshots shown in Fig. 3. Indeed, $K^{*}=1$ corresponds to the fastest evolution and, thus, finite-size effects appear before. For the other two cases the algebraic regime extends for much longer times, and finite-size effects are not found. We obtain that while for $K^{*}=1$ and $K^{*}=1.4$ both $\sigma_{(10)}$ and $\sigma_{(11)}$ evolve with the same exponent, for $K^{*}=0.6$ the two exponents are clearly different. This is indicative of the existence of anisotropic growth and, as we shall discuss below, it is related to the local accumulation of vacancies at the

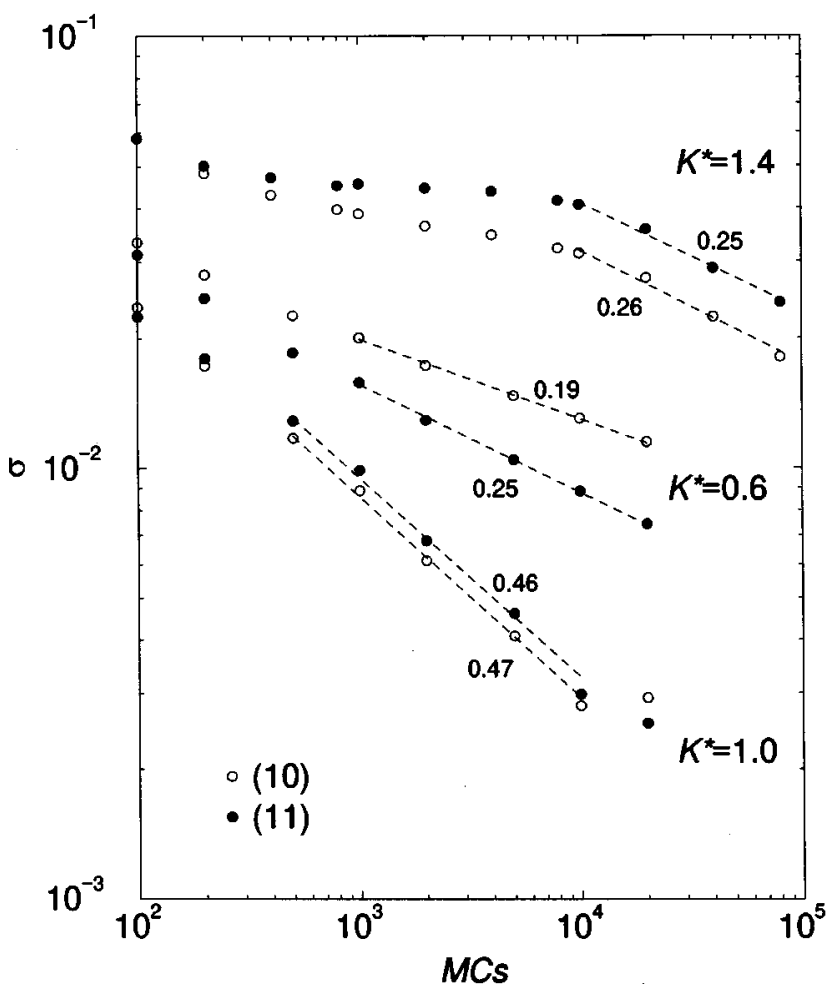

FIG. 4. Width of the structure factor $\sigma$ vs time for $K^{*}=0.6$, $K^{*}=1.0$, and $K^{*}=1.4$. Open circles correspond to the (10) direction and filled circles to the (11) direction. Dashed lines indicate the regions where the growth exponents, written on top, are fitted. 


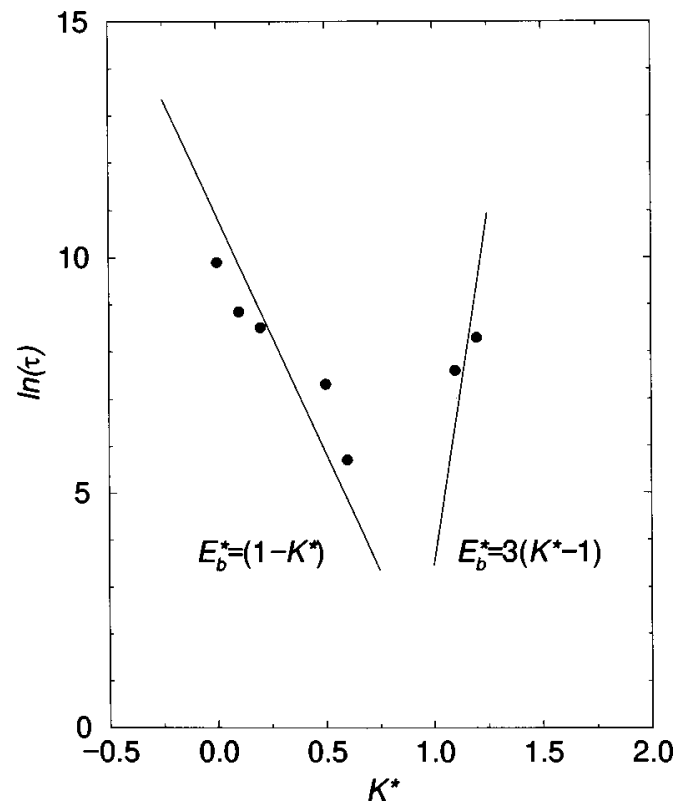

FIG. 5. Time needed to reach the algebraic regime vs $K^{*}$ (black circles). Straight lines are the predicted slopes of these curves from the energy barriers obtained from the model.

vertices of the interfaces (see Fig. 3). Moreover, while the values of the exponents for $K^{*}=1.4$ and $K^{*}=0.6$ are definitively smaller than $1 / 2$, for $K^{*}=1$ it is perfectly consistent with the standard Allen-Cahn value. Notice the long plateau obtained in the case $K^{*}=1.4$. One needs to perform very long simulations before reaching the algebraic growth regime. In fact, we have also obtained such behavior for some values of $K^{*}<1$ inside the region $-0.5<K^{*}<0$. In addition, the extension of the plateau depends on $K^{*}$, suggesting that it is related to the existence of an activated process with energy barriers depending on $K^{*}$. These energies increase (but not symmetrically) as one varies $K^{*}$ from $K^{*}=1$, either to $K^{*}<1$ or to $K^{*}>1$, and in both cases hinder the motion of the vacancies at the vertices of the interfaces. The expressions for the associated energy barriers $\left(E_{b}^{*}=E_{b} / J\right)$ are $E_{b}^{*}=1-K^{*}$ for $K^{*}<1$ and $E_{b}^{*}=3\left(K^{*}-1\right)$ for $K^{*}>1$. In Fig. 5 black dots are the times needed to reach the algebraic regime for the different values of $K^{*}$. These have been estimated from the simulations as the ending points of the plateau. Simultaneously we have plotted (straight lines) the barrier passing time, defined as $\tau \sim \exp \left(E_{b}^{*} / k_{B} T\right)$. As an example, to illustrate how black dots in Fig. 5 have been obtained, we show the case of $K^{*}=0$ (Fig. 6). The evolution up to $\sim 10^{7}$ MCs has been obtained by following first the standard dynamical simulations (circles) and next, by using the $N$-fold way algorithm (squares). The arrow indicates the estimated time for the ending point of the plateau.

We have tested the existence of dynamical scaling. Figures 7(a), 7(b), and 7(c) show the scaled structure factor profiles. Those along the (10) direction have been shifted downwards four decades in order to clarify the picture. Profiles along each direction have been conveniently scaled with the corresponding $\sigma$. The overlap of the data is satisfactory except for the tails at large $q$ 's. Nevertheless, to prove the existence of dynamical scaling it is necessary to have not only the collapse of the curves but also one requires that both

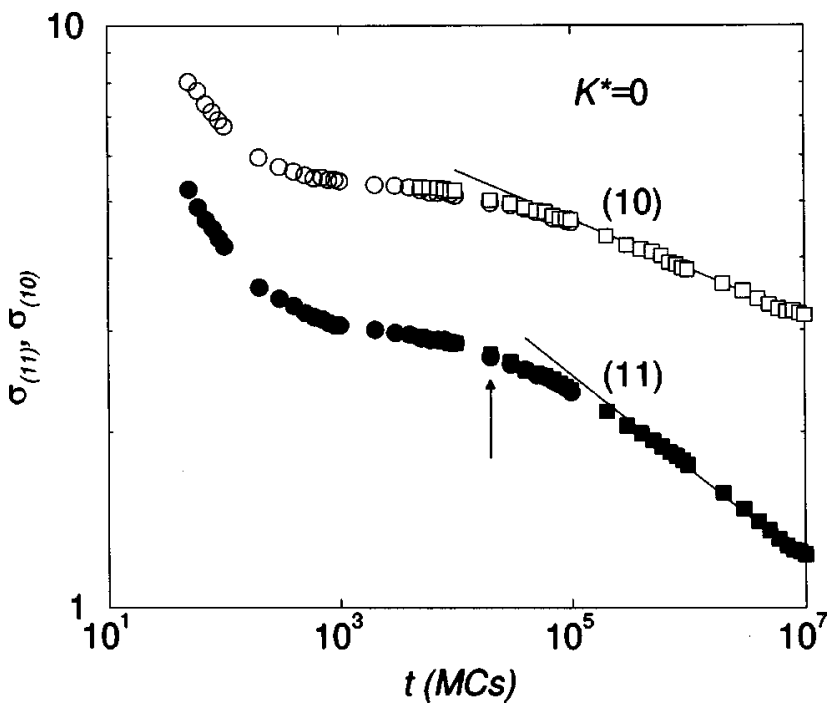

FIG. 6. Structure factor width $\sigma$ vs time for $K^{*}=0$. Open (filled) symbols correspond to the (10) [(11)] direction. The end of the plateau is taken to be at the inflection point, as indicated by the arrow. The results shown by circles have been obtained following standard simulations whereas the long time results, indicated by squares, have been obtained by using the $N$-fold algorithm. The system size is $200 \times 200, c_{V}=0.06$, and $T=0.1 J / k_{B}$.

lengths, $\sigma_{(10)}$ and $\sigma_{(11)}$, evolve with the same power law. This happens to be the case for $K^{*}=1$ and $K^{*}=1.4$, whereas for $K^{*}=0.6$ both sets of profiles scale independently according to widths evolving with different power laws.

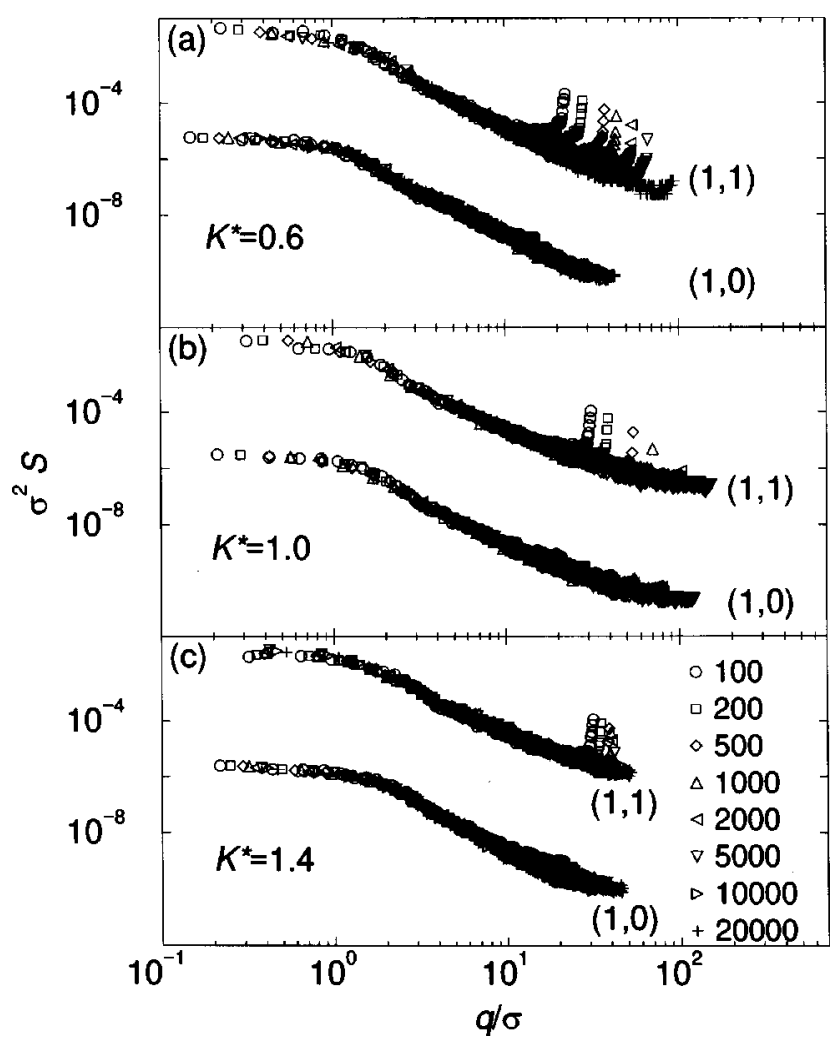

FIG. 7. $\log$-log plot of the scaled structure factor profiles in the (10) and (11) directions for $K^{*}=0.6, K^{*}=1.0$, and $K^{*}=1.4$. The profiles in the (10) direction have been shifted four decades below in order to clarify the picture. 


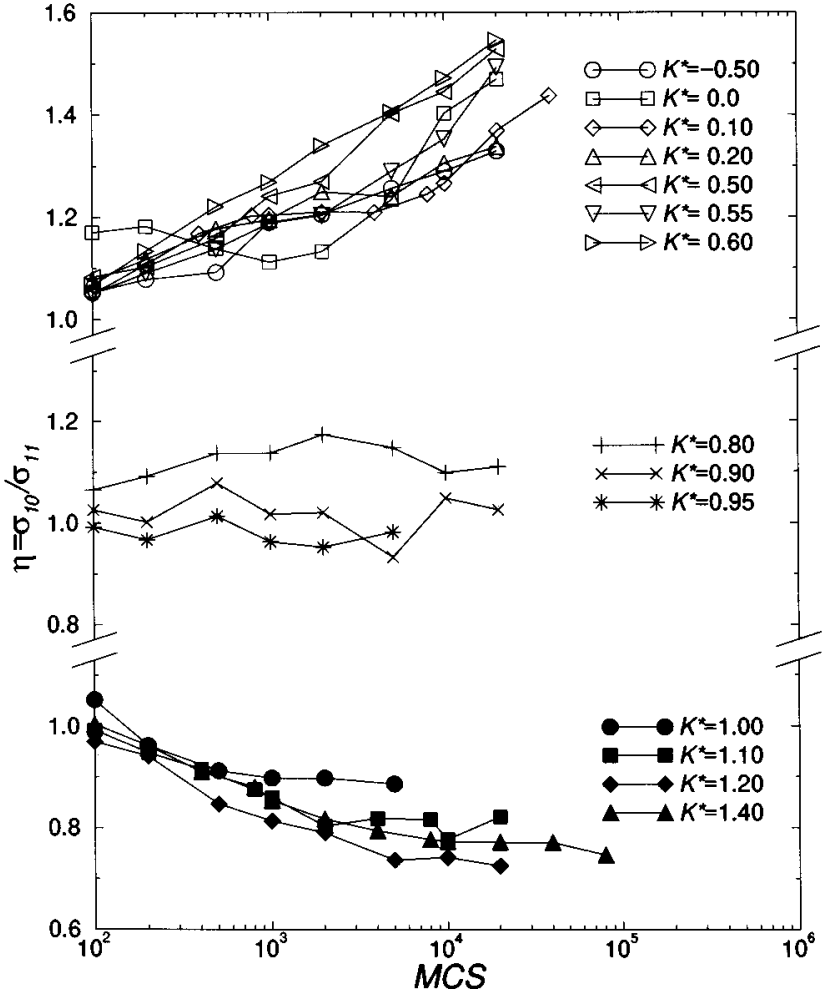

FIG. 8. Ratio $\eta \equiv \sigma_{(10)} / \sigma_{(11)}$ vs time for different values of the parameter $K^{*}$. All these simulations are performed in a $200 \times 200$ square lattice with $c_{V}=0.06$ at $T=0.1 J / k_{B}$.

In the following two figures we present a complete study of both the anisotropic character of the growth and the kinetic growth exponent(s) for a wide range of values of the interaction parameter $K^{*}$. Figure 8 shows the time evolution of the ratio $\eta \equiv \sigma_{(10)} / \sigma_{(11)}$ for different values of $K^{*}$. For $K^{*}<0.8, \eta$ definitively increases with time, showing that the shape of the ordered domains becomes more and more spikelike. For $0.8<K^{*}<1.0$ the ratio remains constant around $\eta=1$, indicating that the domains are circular during all the evolution. For $K^{*}>1$ after an initial decrease, $\eta$ reaches the value $1 / \sqrt{2}$, indicating that, at long times, the domains are squarelike and grow isotropically. Figure 9 shows the growth exponents obtained by fitting an algebraic growth law $\sigma(t) \sim t^{n}$ to the evolution of both $\sigma_{(10)}$ (open circles) and $\sigma_{(11)}$ (filled circles). Note that the Allen-Cahn value $(n \simeq 0.5)$ is only reached for values of $K^{*} \simeq 1$.

We have also studied the behavior of the structure factor at large $q$ 's. It is affected by two different phenomena. (i) Along the direction $(1,1)$ for $q>0.5$ the structure factor is distorted due to the existence of the nonscaling fundamental peak at $\vec{k}=(00)$ [strictly speaking the value of the structure factor at $\vec{k}=(00)$ is always zero, but the peak exhibits some finite width due to the existence of disorder in the system]. (ii) For the cases in which the vacancies dissolve into the bulk, there is a homogeneous nonscaling background that, in turn, may evolve in time.

\section{DISCUSSION}

The differences in the values obtained for the kinetic exponent of the growth law lie on the different characteristics

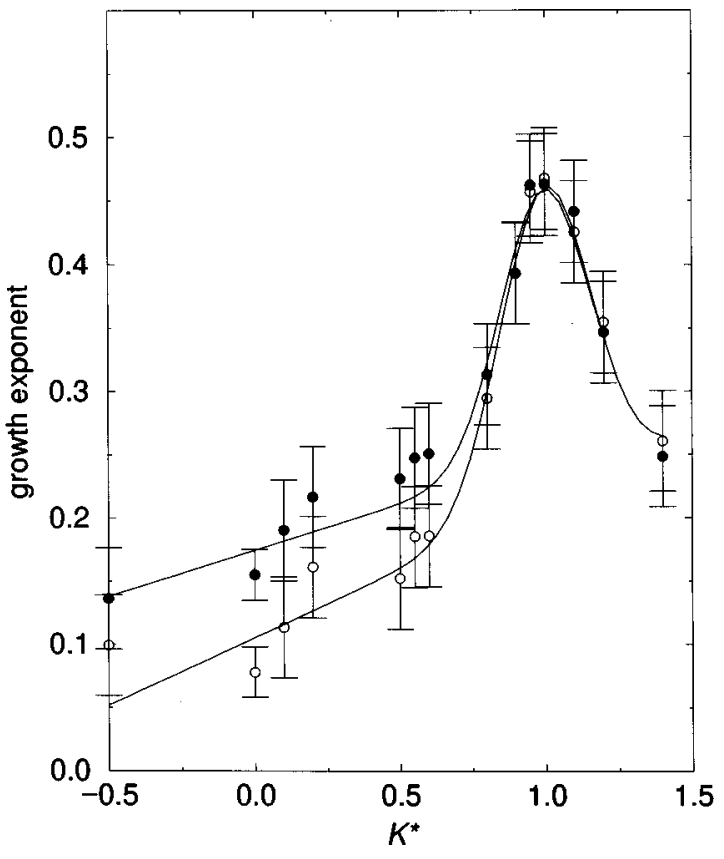

FIG. 9. Growth exponent vs $K^{*}$ obtained from the structure factor width $\sigma$. Open (filled) circles correspond to the evolution of $\sigma_{(10)}\left(\sigma_{(11)}\right)$. All the simulations are performed in a $200 \times 200$ square lattice with $c_{V}=0.06$ at $T=0.1 J / k_{B}$.

of the intercoupling between bulk diffusing vacancies and the interface motion. For $K^{*} \neq 1$ the intercoupling proceeds via an effective interaction originated from the vacancyvacancy specific interaction. Moreover, this introduces differences in the internal structure of the interface. In the particular case of $K^{*}=1$, this intercoupling reduces to a simple encounter between curvature-driven interfaces and mobile vacancies that mutually cross their respective trajectories. This does not make the curvature ineffective but may slow down the domain growth. It has been shown that ${ }^{8}$ the effect of this simple intercoupling does not modify the essential time dependence of the growth law but modifies the growth rate (prefactor) that decreases as the mobile impurity concentration increases.

We next discuss separately the other two cases. We start with the case of vacancy attraction $\left(K^{*}=0.6\right)$ and point out some other relevant features present in Fig. 3. Notice the increasing concentration of vacancies at the interfaces as they evolve with time. This is premonitory of the phase separation process, eventually reachable at longer times. During the regime shown in Fig. 3 one is mainly concerned with a nonhomogeneous distribution of vacancies along the interfaces. The local accumulation at the vertices deserves special mention. This is the signature of a previous fast process of interface reduction (due to the high curvature of the vertices). The further evolution is hindered until the vacancies diffuse along the interfaces. This involves activated processes. Indeed, in our simulations we have observed how the temporal pinning of the high-curvature portion of the interfaces provokes that the further evolution of the interconnecting interfaces (with lower curvature) does not fulfill the main assumptions underlying the Allen-Cahn theory. The importance of this temporal pinning depends on $K^{*}$, since the energy barriers $\left(E_{b}^{*}=1-K^{*}\right)$ hindering the motion of a 
$t(M C s)$ $10^{6}$

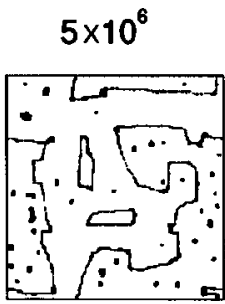

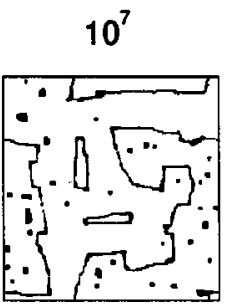

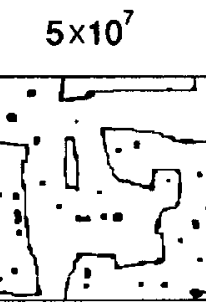

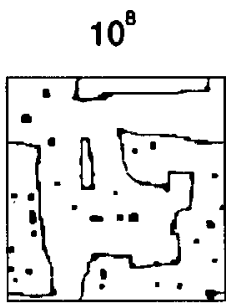

FIG. 10. Snapshots of some evolving domains directly extracted from our simulations for $K^{*}=0$. Vacancies ( $A$ and $B$ particles) are shown as black (white). The simulations are performed in a $200 \times 200$ square lattice with $c_{V}=0.06$ at $T=0.1 J / k_{B}$. vacancy at the corner of the interface increase as $K^{*}$ decreases from $K^{*}=1$. In Fig. 10 we show this effect for the case $K^{*}=0$. In particular, one observes how the single (rectangular) domains evolve so that they become more and more platelike. This is because the longer interfaces (with a lower concentration of vacancies) are the only ones able to evolve. Moreover, they remain (almost) flat and parallel to the (10) direction. This feature is not observed in previous studies of the diluted antiferromagnetic Ising model ${ }^{14}$ (notice that it corresponds to the BEG with $K^{*}=0$ ) so that it cannot be attributed exclusively to the interaction (which favors the vacancies to be nearest neighbors at the interface). In Fig. 11 we schematically illustrate this mechanism of evolution. This represents a typical domain in the regime under discussion here, that is, the regime characterized because the width of the interface is small and the only relevant length is the size of the $\mathrm{AB}$ ordered domains. Two facts have to be taken into account: the attractive vacancy-vacancy interaction defined by the Hamiltonian and the conserved character of the implemented dynamics (Kawasaki). The combination of both introduces energy barriers hindering the motion of the vacancies (circles) at the interfaces, which hinders the shrinkage of the domain. In Fig. 11 we have indicated in black (white) the vacancies with associated energy barriers so that they are induced to go outward (inward). The vacancies in gray do not have any preference. The crucial point is that the inwards motion of the vacancies at the corner is strongly hindered whereas for its neighbors, it is favored. This provokes that the shrinkage of the domains proceeds by displacing the flat interfaces and accumulating the excess vacancies at the vertices. ${ }^{26}$ The domains then become spikelike along the (11) direction, breaking down the single-length dynamical scaling so that the growth becomes anisotropic. In [Ref. 14], the authors coupled the Glauber dynamics for the spins to the conserved dynamics for the number of vacancies in such a way that the vacancy at the corner is not pinned. Nevertheless they found that the dynamical evolution of the ordering process is effectively described by a logarithmic growth law (in the limit of low vacancy concentration). Other studies ${ }^{13}$ on the diluted ferromagnetic Ising model, encountered that, by coupling both dynamics differently (the simultaneous vacancy-spin exchange and spin flip is not allowed) so that the vacancy at the corner is effectively pinned, the growth stops. In the case of the alloy, the dynamics implemented follows directly from the requirement of the conservation law for the number of particles and we found that the ordering process is definitively described by a growth law that, although slower than the Allen-Cahn law, is definitively algebraic. For some values of $K^{*}$ this algebraic regime is preceded by a plateau whose extension in time depends on $K^{*}$. More precisely, it is longer the bigger the energy barriers are. In particular, we found that for $K^{*}=0$ this algebraic regime is visible only after $\sim 10^{5} \mathrm{MCs}$ (see Fig. 6). We notice that the BEG model, in the particular case of $K=L=0$, corresponds to a diluted Ising model. In view of this, we believe that the growth law for the diluted antiferromagnet is algebraic. In fact, the authors of [14] did not exclude this possibility in their discussion. Concerning the complete pinning of the process reported by [13], it is certainly due to the extremely low value of the temperature.

Later on, as the width of the interface increases, this pinning effect, localized at the corner, becomes less important and one expects the system to cross over to a completely different regime. In this asymptotic regime, not reached in our simulations, discussing the interface, as formed by the increasing accumulation of vacancies, is meaningless. Rather, one would deal with a phase-separation process, which is not studied in the present work.

We now focus on the discussion for $K^{*}=1.4$. In this case, the repulsive interaction between vacancies favors them to be next nearest neighbors at the interfaces. As previously, the driving force is contained in the corner but its motion is hindered by a barrier of energy $2\left(K^{*}-1\right)$. The interface connecting two vertices is directed along the (11) direction and evolves so that it displaces in a parallel manner. The energy barrier associated to this mechanism is (a)

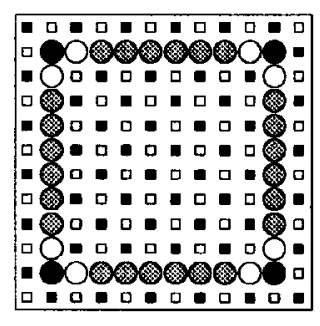

(b)

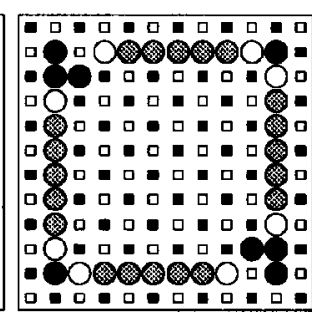

(c)

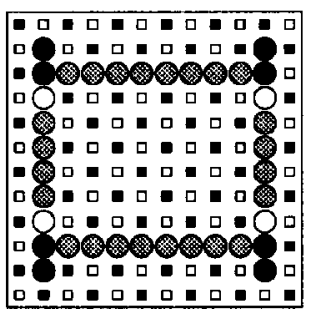

(d)

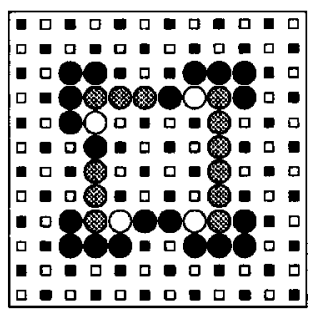

FIG. 11. Schematic representation of the evolution of a square domain typically observed in the $K^{*}<1$ simulations. The circles represent vacancies whereas the white (black) squares represent $A$ (B) particles. The mechanism of evolution is emphasized by showing in black (white) those vacancies that have the tendency to move outward (inwards). The vacancies painted in grey are the ones that are, in this sense, indifferent. 


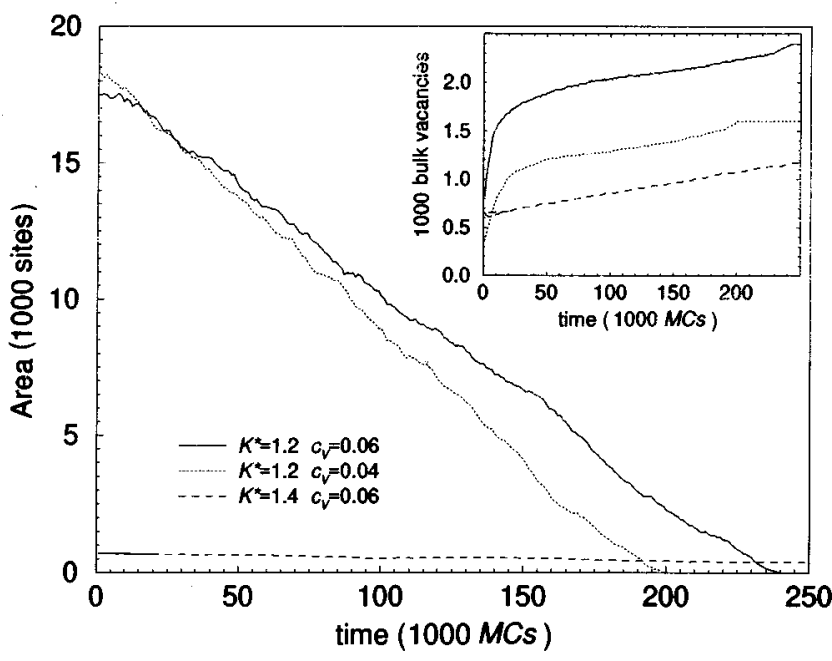

FIG. 12. Domain area vs time of a single domain for $K^{*}=1.2$ (solid and dotted lines) and $K^{*}=1.4$ (dashed line). The solid (dotted) line corresponds to $c_{V}=0.06\left(c_{V}=0.04\right)$. We simultaneously show, in the inset, the time evolution of the total amount of bulk vacancies in the system. The simulations are performed in a 200 $\times 200$ square lattice at $T=0.1 J / k_{B}$.

$E_{b}^{*}=3\left(K^{*}-1\right)$. The vacancies, in excess as a consequence of the interface reduction, are in this case (vacancy repulsion) expelled to the bulk. Moreover, the migrating interface has to cope with the effective repulsive interaction due to the bulk vacancies, whose concentration increases as the system evolves. Indeed, one expects this should interfere with the dynamics. At this point, it is interesting to discriminate whether or not this interference makes the curvature-driven mechanism become ineffective. In this sense, we have verified that the interface evolves covering a domain area constant in time. This is shown if Fig. 12 for single domains directly extracted from our simulations. These calculations have been performed using the optimized multigrid algorithm discussed previously. A linear time dependence for the domain area evolution is obtained. This is commonly accepted as indicative that the motion of the interface is curvature driven. ${ }^{27}$ It then follows that the effect of the intercoupling between mobile bulk vacancies and the evolving interfaces is, in this case, to slow down the global process as it is revealed by a decreasing in the effective growth exponent but it does not make ineffective the curvature driven mechanism, which remains as the underlying mechanism for the motion of the interfaces. Moreover the effective exponent tends to the ideal 1/2 Allen-Cahn value as $K^{*} \rightarrow 1$.

\section{SUMMARY}

We use a Blume-Emery-Griffiths model (with $L=0$ ) to study the influence of mobile vacancies on the kinetics of domain growth in a stoichiometric binary alloy after quenches to a very low temperature $\left(T=0.1 J / k_{B}\right)$ through an order-disorder transition. The study is performed by Monte Carlo simulations in the limit of low vacancy concentration for a wide range of values of the biquadratic coupling parameter $K^{*}$, which controls the specific vacancy-vacancy interaction. For all values of $K^{*}$ inside the range $-0.5 \leqslant K^{*} \leqslant 1.4$ we found that the vacancies tend to concentrate at the interfaces. This feature introduces, via the parameter $K^{*}$, an intercoupling between diffusing bulk vacancies and moving interfaces. In the particular case of $K^{*}=1$ this intercoupling does not take place via any specific interaction and the ordering process is consistent with the Allen-Cahn law. In fact we find that $n \sim 1 / 2$ for $K^{*} \sim 1$ whereas the exponent is clearly smaller when such interaction is present, no matter if it is attractive $\left(K^{*}<1\right)$ or repulsive $\left(K^{*}>1\right)$. Nevertheless, our results clearly show that the growth is definitively algebraic. When $K^{*}<1$ the attractive vacancy interaction favors the increasing accumulation of vacancies at the interfaces as the system evolves. The regime of interest here corresponds to the very initial stages of a phase separation process when the width of the interfaces is small and the only relevant length is the size of the $A B$ ordered domains. Our main finding is that for quenches inside the coexistence region the growth for the binary alloy is, in this regime, anisotropic. This is related to the existence of energy barriers (depending on $K^{*}$ ) that hinder the motion of the vacancies at the vertices of the (10) squarelike domains and simultaneously provoke local accumulations of vacancies along the (11) directions. Furthermore, these barriers may delay the apparition of the algebraic regime of the growth process to very late times. Concerning the underlying mechanism for the motion of the interfaces, it is not purely curvature driven and the two effective exponents needed to describe the process are lower than the Allen-Cahn value. Nevertheless, both tend to $n=1 / 2$ as $K^{*} \rightarrow 1$. For $K^{*}>1$ the specific vacancyvacancy interaction is repulsive and the interfaces of the square-like domains are in this case directed along the (11) directions. As in the previous case, the motion of the vacancies at the vertices is an activated process. The associated energy barriers depend on $K^{*}$ and the algebraic growth regime shows up only after the time needed for surpassing the barrier. Since the interface motion has to cope with the repulsive interaction with diffusing vacancies, we found that the ordering process clearly slows down. Nevertheless, this repulsion does not make the curvature ineffective. The effective exponent is lower than the Allen-Cahn value but approaches $n=1 / 2$ as $K^{*} \rightarrow 1$, as expected.

\section{ACKNOWLEDGMENTS}

We acknowledge financial support from the Comisión Interministerial de Ciencia y Tecnología (CICyT, project number MAT95-504) and supercomputing support from Fundació Catalana per a la Recerca (F.C.R.) and Centre de Supercomputació de Catalunya (CESCA). M.P. and C.F. also acknowledge financial support from the Comissionat per a Universitats i Recerca (Generalitat de Catalunya). 
${ }^{1}$ Dynamics of Ordering Processes in Condensed Matter, edited by S. Komura and H. Furukawa (Plenum, New York, 1988).

${ }^{2}$ J.D. Gunton, M. San Miguel, and P.S. Sahni, in Phase Transitions and Critical Phenomena, edited by C. Domb and J.L. Lebowitz (Academic, New York, 1983), Vol. 8.

${ }^{3}$ S.M. Allen and J.W. Cahn, Acta Metall. 27, 1085 (1979).

${ }^{4}$ I.M. Lifshitz and V.V. Slyozov, J. Phys. Chem. Solids 19, 35 (1961).

${ }^{5}$ M.K. Phani, J.L. Lebowitz, M.H. Kalos, and O. Penrose, Phys. Rev. Lett. 453661980.

${ }^{6}$ P.S. Sahni, G. Dee, J.D. Gunton, M. Phani, J.L. Lebowitz, and M. Kalos, Phys. Rev. B 24, 410 (1981).

${ }^{7}$ H.C. Fogedby and O.G. Mouritsen, Phys. Rev. B 37, 5962 (1988), and references therein.

${ }^{8}$ M. Porta and T. Castán, Phys. Rev. B 54, 166 (1996).

${ }^{9}$ F. Bley and M. Fayard, Acta Metall. 24, 575 (1976).

${ }^{10}$ R.F. Shannon, C.R. Harkless, and S.E. Nagler, Phys. Rev. B 38, 9327 (1988).

${ }^{11}$ D.A. Huse and C.L. Henley, Phys. Rev. Lett. 54, 2708 (1985).

${ }^{12}$ H. Gilhøj, C. Jeppesen, and O.G. Mouritsen, Phys. Rev. E 52, 1465 (1995).

${ }^{13}$ D.J. Srolovitz and G.N. Hassold, Phys. Rev. B 35, 6902 (1987).

${ }^{14}$ O.G. Mouritsen and P.J. Shah, Phys. Rev. B 40, 11445 (1989); P.J. Shah and O.G. Mouritsen, ibid. 41, 7003 (1990).

${ }^{15}$ O.G. Mouritsen, P.J. Shah, and J.V. Andersen, Phys. Rev. B 42, 4506 (1990).
${ }^{16}$ K. Oki, H. Sagane, and T. Eguchi, J. Phys. (France) Colloq. 38, C7-414 (1977).

${ }^{17}$ T. Ohta, K. Kawasaki, and A. Sato, Phys. Lett. A 126, 93 (1987).

${ }^{18}$ H. Gilhøj, C. Jeppesen, and O.G. Mouritsen, Phys. Rev. E 53, 5491 (1996).

${ }^{19}$ H. Gilhøj, C. Jeppesen, and O.G. Mouritsen, Phys. Rev. Lett. 75, 3305 (1995).

${ }^{20}$ S.E. Nagler, R.F. Shannon, C.R. Harkless, M.A. Singh, and R.M. Nicklow, Phys. Rev. Lett. 61, 718 (1988).

${ }^{21}$ M. Blume, V.J. Emery, and R.B. Griffiths, Phys. Rev. A 4, 1071 (1971).

${ }^{22}$ E. Vives and A. Planes, Phys. Rev. Lett. 68, 812 (1992); Phys. Rev. B 47, 2557 (1993); C. Frontera, E. Vives, and A. Planes, ibid. 48, 9321 (1993).

${ }^{23}$ Details of the mean-field calculation, using the self-consistent field method, will be given elsewhere.

${ }^{24}$ G. Porod, in Small Angle X-Ray Scattering, edited by O. Glatter and L. Kratky (Academic, New York, 1982).

${ }^{25}$ A.B. Bortz, M.H. Kalos, and J.L. Lebowitz, J. Comput. Phys. 17, 10 (1975).

${ }^{26}$ If this analysis is done in a circular domain, it can be seen that it first evolves to a square domain and then proceeds as discussed.

${ }^{27}$ E. Domany and D. Kandel, in Cellular Automata and Modeling of Complex Physical Systems, edited by P. Manneville, N. Boccara, G.Y. Vichniac, and R. Bidaux (Springer-Verlag, Berlin, 1990), p. 98 . 